\title{
Bernard ÉLIE
}

Économiste, Université du Québec à Montréal

\author{
février 2009
}

\section{"L’origine de la crise."}

Un document produit en version numérique par Jean-Marie Tremblay, bénévole, professeur de sociologie au Cégep de Chicoutimi

Courriel: jmt_sociologue@videotron.ca

Site web: http://www.uqac.ca/jmt-sociologue/

Dans le cadre de la collection: "Les classiques des sciences sociales"

Site web: http://www.uqac.ca/Classiques_des_sciences_sociales/

Une collection développée en collaboration avec la Bibliothèque

Paul-Émile-Boulet de l'Université du Québec à Chicoutimi

Site web: http://bibliotheque.uqac.uquebec.ca/index.htm 


\section{Politique d'utilisation de la bibliothèque des Classiques}

Toute reproduction et rediffusion de nos fichiers est interdite, même avec la mention de leur provenance, sans l'autorisation formelle, écrite, du fondateur des Classiques des sciences sociales, Jean-Marie Tremblay, sociologue.

Les fichiers des Classiques des sciences sociales ne peuvent sans autorisation formelle:

- être hébergés (en fichier ou page web, en totalité ou en partie) sur un serveur autre que celui des Classiques.

- servir de base de travail à un autre fichier modifié ensuite par tout autre moyen (couleur, police, mise en page, extraits, support, etc...),

Les fichiers (.html, .doc, .pdf, .rtf, .jpg, .gif) disponibles sur le site Les Classiques des sciences sociales sont la propriété des Classiques des sciences sociales, un organisme à but non lucratif composé exclusivement de bénévoles.

Ils sont disponibles pour une utilisation intellectuelle et personnelle et, en aucun cas, commerciale. Toute utilisation à des fins commerciales des fichiers sur ce site est strictement interdite et toute rediffusion est également strictement interdite.

\section{L'accès à notre travail est libre et gratuit à tous les utilisateurs. C'est notre mission.}

Jean-Marie Tremblay, sociologue

Fondateur et Président-directeur général, LES CLASSIQUES DES SCIENCES SOCIALES. 
Cette édition électronique a été réalisée par Jean-Marie Tremblay, bénévole, professeur de sociologie au Cégep de Chicoutimi à partir de :

Bernard Élie, économiste québécois

\section{“L’origine de la crise”}

Un article publié en deux parties sur le site ÉCONOMIE autrement.org,. $1^{\mathrm{re}}$ partie : L’origine, le pourquoi (10 février 2009). $2^{\mathrm{e}}$ partie : Les sorties de crise. (22 février 2009).

Bernard ÉLIE est professeur au département de sciences économiques de l'UQAM. Spécialiste des théories monétaires et de l'économie financière internationale, il est aussi vice-doyen de la Famille sciences humaines de l'UQAM. Ses publications portent sur les transformations du système financier international.

[L'auteur nous a accordé, le 26 février 2010, sa permission de rediffuser ce texte dans Les Classiques des sciences sociales.]

Courriel : elie.bernard@uqam.ca

Polices de caractères utilisée :

Pour le texte: Times New Roman, 12 points.

Pour les citations : Times New Roman 12 points.

Pour les notes de bas de page : Times New Roman, 12 points.

Édition électronique réalisée avec le traitement de textes Microsoft Word 2008 pour Macintosh.

Mise en page sur papier format

LETTRE (US letter), 8.5'” x 11’')

Édition complétée le 27 février 2010 à Chicoutimi, Ville de Saguenay, Québec. 


\section{Table des matières}

$\underline{1}^{\text {re }}$ partie. L'origine, le pourquoi. (10 février 2009).

$\underline{2}^{\mathrm{e}}$ partie. Les sorties de crise. (22 février 2009). 
Bernard ÉLIE

“L’ORIGINE DE LA CRISE.”

Un article publié en deux parties sur le site ÉCONOMIE autrement.org, $1^{\text {re }}$ partie : L'origine, le pourquoi (10 février 2009). $2^{\mathrm{e}}$ partie : Les sorties de crise. (22 février 2009).

\section{$1^{\mathrm{re}}$ partie \\ L'ORIGINE, LE POURQUOI}

(10 février 2009)

\section{ECONOMIEautrement.org}

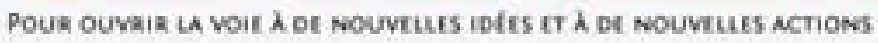

\section{$\underline{\text { Retour à la table des matières }}$}

La récession, fruit des désordres financiers, arrive. La crise qui commence nous a dévoilé, encore une fois, une série de turpitudes : multiplications de titres douteux, falsifications de documents comptables, détournements de fonds, versements de salaires faramineux et de primes exorbitantes. Les organismes de surveillance ont laissé faire ! La sphère financière s'est auto reproduite pendant des années, elle est totalement décrochée du monde réel, de la sphère de production, qu'elle a déstabilisé en provoquant une grave récession. La cupidité des grands financiers n'a pas de limite. Après une année 2008 catastrophique, le petit monde de Wall Street s'est quand même versé 18,4 milliards de dollars de primes pour l'année qui vient de se terminer. Des milliers de milliards ont été perdus et les États ont dû allonger des sommes gigantesques pour sauver les institutions financières et pour limiter l'ampleur des dégâts dans l'économie réelle qui ne sera pas épargnée. 
Comment en être arrivé là ?

\section{L'origine}

L’arrivée au pouvoir de Margaret Thatcher, puis de Ronald Reagan, a consacré le retour des idées libérales soutenues par Milton Friedman. « L’ÉTAT N’EST PAS LA SOLUTION. IL EST LE PROBLEME » disait Reagan pendant sa première campagne électorale contre Jimmy Carter. Ce slogan traduit bien les renversements économiques qui vont suivre.

L’épuisement du modèle établi après-guerre se résume en trois expressions : inflation, chômage (la stagflation) et baisse de la productivité du travail. La nouvelle politique économique libérale reposera d'abord sur la tentative de contrôler la masse monétaire et sur des taux d'intérêt élevés, pour lutter contre l'inflation, mais surtout pour limiter la dévalorisation des actifs financiers. L’objectif sera aussi le transfert de la richesse vers les investisseurs et les entreprises ${ }^{1}$ pour relancer la productivité.

La réduction durable des impôts sur les revenus, pour ceux qui en payent, est toujours la mesure prioritaire des politiques libérales pour assurer la croissance. Cette priorité est celle des républicains aux États-Unis, de Reagan à G. W. Bush, et des conservateurs canadiens. De telles baisses ont un double avantage à leurs yeux : permettent aux plus riches d'investir et d'obliger l'État à restreindre à terme ses programmes pour respecter la règle d'un budget équilibré.

Pour assurer le succès de cette politique, la seule vraiment crédible pour eux, il faudra réduire les réglementations trop contraignantes, pour permettre ainsi au marché de nous conduire le plus librement possible à l'optimum. L'État doit être minimum.

1 De 1980 à 2005, le salaire médian au Canada n’a augmenté que de 0,1\%, en terme réel, alors que le salaire moyen a connu une hausse de 55\%. Lorsque le salaire moyen progresse plus que le salaire médian, cela indique l'accentuation des inégalités. Voir Statistique Canada, nº 97-563-X au catalogue, mai 2008. 


\section{La déréglementation financière}

L'effondrement de l'Accord de Bretton Woods (de facto en 1968, de jure en 1976) inaugure l'ère de l'instabilité des taux de change et des taux d'intérêt et de la croissance des risques. Face à ce nouveau contexte, les établissements financiers vont réagir. La première parade sera de réclamer la diversification de leurs activités. Partout, le système financier sera décloisonné, déréglementé. Par exemple au Canada, les banques pourront faire du courtage, de la fiducie et de l'assurance. De simples banques, elles se transforment en groupes financiers.

La deuxième parade sera d'exiger la libéralisation des mouvements des capitaux ce qui donnera accès à un plus grand nombre de marchés et à plus de choix d’investissements. Amorcé en Angleterre et aux États-Unis, le mouvement se généralisera dans les années 1990. Cette volonté de libéralisation des flux de capitaux conduira à l’Accord de libre-échange Canada-États-Unis (1987), premier accord international portant sur la liberté de circulation des capitaux. Cet accord sera le modèle à généraliser. D’abord en incluant le Mexique (ALENA), puis dans l'élaboration de l'AMI (l'Accord multilatéral sur les investissements) à l’OCDE, dans les années 1990, qui fut bloqué. Mais il ne tardera pas à s’installer discrètement par différents moyens plus effacés qu'un grand accord mondial. La domination de la sphère financière est consacrée.

\section{La table est mise pour la crise}

Les tenants du tout au marché triomphent, le nirvana est proche. Les déficits publics sont bannis, des lois sur le déficit-zéro sont adoptées à travers la planète, la libre circulation des biens et des services s'impose et les capitaux peuvent maintenant aller là où ils peuvent maximiser les rendements. Il faut que le système tourne, les crises épisodiques ne sont que de mauvais moments à passer. Certaines crises financières sont assez dures (en Asie du Sud-est en 1998) et plusieurs krachs boursiers font très mal (comme celui des nouvelles technologies en 2001), mais les pertes sont limitées dans le temps et dans l'espace. Il ne reste plus qu'à augmenter la consommation, quit- 
te à accroître l'endettement. Les années 2000 seront bénies. C'est la fin de la sousconsommation, toute relative, des années 1980 et 1990. Les faibles revenus, signe de la croissance des inégalités, seront-ils des freins ? Non, augmentons la consommation et élargissons le crédit.

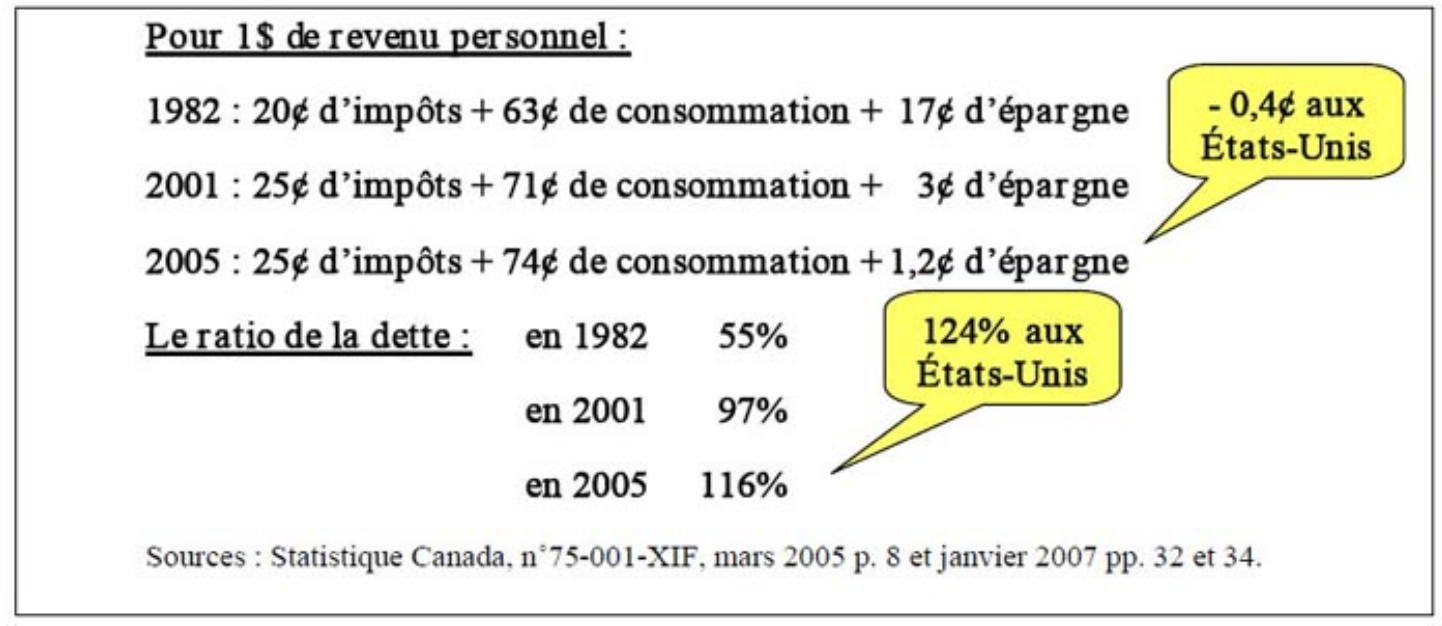

Canada l'épargne passa de 17\% du revenu disponible en 1982 à 1,2\% en 2005. Le ratio de la dette (dette totale/revenu disponible) augmenta à $116 \%$ en 2005. La consommation fut croissante, mais avec des revenus stables et de plus en plus inégalitaires l'endettement devait croître en conséquence. La population est donc de plus en plus vulnérable aux interruptions de revenu, plus fragile en cas de crise. Les taux d'intérêt très bas, initié par la Réserve fédérale américaine, va encourager l'endettement.

\section{L'occasion fait le larron}

La bulle immobilière des dernières années va enhardir les établissements financiers à profiter, à court terme, de cette belle occasion. Grâce aux gains de capitaux que peut engendrer la hausse des prix dans l'immobilier, élargissons le nombre des emprunteurs. Même pour des clients peu solvables, c'est-à-dire à risque élevé, il est possible de leur accorder un prêt hypothécaire reposant sur des hausses de prix des immeubles qui semblent sans fin. Les «surprimes » sont nées. Les établissements financiers pour s'assurer un niveau de liquidités suffisant, émettront des titres à court 
terme, les papiers commerciaux, appuyés, garantis sur ces actifs que sont les hypothèques à haut risque. Ces papiers commerciaux achetés aux quatre coins de la planète, vont transmettre le virus partout, des risques plus élevés.

En 2007, la bulle immobilière s’arrêta et puis se dégonfla. Les emprunteurs devenus insolvables firent défaut ( $\pm 10 \%$ du marché), ils ne pouvaient plus répondre à leurs engagements. Ce qui entraîna la dévalorisation des hypothèques accordées et des titres adossés à elles, comme les fameux papiers commerciaux. Tous furent atteints. N'étant pas remboursés le manque de liquidités se généralisa dans les établissements financiers. Leur premier réflexe fut de limiter, de resserrer les crédits accordés aux entreprises et aux particuliers. Sans crédit, la chaîne de production est rompue. Le capitalisme sans crédit n’a plus de lubrifiant et arrête de produire. Les mises à pied vont amplifier le ralentissement qui alors se généralise. La crise est là. Depuis un an, les gouvernements, directement ou par l'intermédiaire de leur Banque centrale, ont injecté des masses énormes de liquidités dans les banques pour faire repartir la machine, mais sans succès.

Les vœux du G20 (le 15 novembre dernier), les programmes de relance des présidents Barack Obama et Nicolas Sarkozy, de la Chancelière Angela Merkel et du Premier ministre Gordon Brown permettront-ils de sortir de la crise ? Nous y répondrons au prochain texte : II - La crise et sa sortie. Au Canada, le piètre budget de Jim Flaherty aura bien peu de résultats, la sortie de crise du Canada viendra avec la reprise américaine. 
$2^{\mathrm{e}}$ partie

\section{LES SORTIES DE CRISE}

(22 février 2009)

\section{ECONOMIEautrement.org}

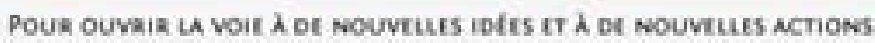

$\underline{\text { Retour à la table des matières }}$

Dans le premier texte, «L'origine, le pourquoi » de la crise, je vous annonçais un second texte sur « la crise et sa sortie », en réalité nous devons mettre le pluriel pour être conforme à la réalité.

Pour certains, il faudrait se réjouir de la crise car elle permet d'entrevoir la fin du capitalisme et la création d'un nouveau monde. Les coûts sociaux et la détresse humaine seraient tels qu'un tel scénario est inenvisageable. Le grand soir n’arrivera pas, comme un nouveau monde. D’ailleurs, cette démarche rejoint celle des républicains américains, ne rien faire, sauf de réduire les impôts. Pour ces derniers, l’économie sera ainsi purgée de ses mauvais éléments et le marché, laissé libre, nous conduira au meilleur des mondes. Dans ces deux cas, les perdants seront les plus pauvres sans protection et les travailleurs les plus fragiles. Les plus riches sauront bien se tirer d'affaire. C'est le cas de le dire ! 


\section{A - LA CRISE FINANCIÈRE : LA STRATÉGIE DES PAYS INDUSTRIALISÉS}

\section{1 - Relancer le crédit}

La conséquence de l'expansion des mauvaises créances au bilan des établissements financiers fut d'abord l'arrêt des prêts entre ces établissements. Puis, le flux des crédits vers les entreprises et les particuliers se tarit. Au fur et à mesure que la crise financière prenait de l'ampleur, les interventions des autorités dans le système financier vont se faire de plus en plus directement.

\section{1 - Baisse des taux d'intérêt}

La Banque du Canada, depuis 15 mois, a réduit ses taux d’intérêt. Son taux cible, souvent appelé le taux directeur, est passé de 41/2 \% à $1 \%$ (le 20 janvier dernier). Cette stratégie, plus accentuée depuis octobre 2008, visait à réduire les coûts d'emprunt et à faciliter aux banques l'accès aux liquidités de la banque centrale. L’objectif était de relancer le crédit par une plus grande abondance de liquidités. Mais les banques ont peu suivi. La poursuite de la baisse de la qualité de leur actif a augmenté leurs craintes de pertes devant des clients de moins en moins solvables. Ce risque d’illiquidité conduira les banques à être plus parcimonieuses dans les prêts accordés.

\section{2 - Fournir des liquidités au système financier}

Pour inciter les banques à changer de comportement, les autorités monétaires canadiennes mirent à la disposition des banques des liquidités supplémentaires. Espérant ainsi réduire leur peur de manquer de liquidités pour répondre à leurs obligations. Les banques centrales achetèrent des titres possédés par les banques privées en contrepartie de liquidités. Ces opérations ont pour nom prises en pension, puisque ces échanges sont limités dans le temps. Au 31 décembre dernier, le total des liquidités mises ainsi à la disposition des banques par ce moyen était de 37,2 milliards, contre 4 
milliards un an auparavant ${ }^{2}$ et zéro au printemps 2007. Mais les banques sont restées aussi craintives de manquer de liquidités, elles ont même augmenté certains taux d’intérêt pour diminuer les emprunteurs possibles.

Les autorités canadiennes ont aussi mis en place le Programme d'achat de prêts hypothécaires assurés, pour un total de 125 milliards (75 milliards dans un premier temps et 50 milliards lors du dernier budget Flaherty). Les prêteurs, surtout les banques, peuvent donc vendre des hypothèques de très bonne qualité à la Société canadienne d'hypothèques et de logement (SCHL) et obtenir ainsi des liquidités et un financement stable à long terme qui devrait leur permettre de continuer d'accorder du crédit aux entreprises et aux consommateurs canadiens.

Ces deux dernières mesures ne sont que des échanges d'hypothèque ou de titres contre des liquidités provenant de la Banque du Canada, directement ou via la SCHL. L’État fédéral n’a pas ici à augmenter son endettement.

1.3 - Recapitaliser et prêter aux établissements financiers pour en prendre le contrôle

Le Canada n’a pas eu recours à ces expédients. Par contre, en Europe et aux ÉtatUnis ces moyens ont été très utilisés. Il s’agit, pour l’État, de prendre une participation minoritaire ou majoritaire dans le capital d'un établissement financier. L’État en détenant des actions prend le contrôle de ces entreprises ainsi recapitalisées. De même, les prêts accordés à ces entreprises donnent la gouvernance au gouvernement. Dans les deux cas, ces établissements voient leurs liquidités s’accroître. La politique de crédit aux particuliers et aux entreprises, comme la rémunération des cadres, peut donc être revues !

Deux exemples : ainsi, en septembre dernier, la Banque d'Angleterre accorda un prêt d'urgence à la banque Northern Rock qui fut par la suite nationalisée par le gouvernement britannique. Aux États-Unis, la Réserve fédérale (la Fed) et le gouvernement de George W. Bush décidèrent de sauver l'assureur AIG (American International Group) menacé de faillite en lui apportant une aide de 85 milliards \$US en échange de $79,9 \%$ de son capital, de ses actions.

2 Statistiques bancaires et financières de la Banque du Canada, janvier 2009, tableau B1, p. S10. 
Ces opérations sont financées par des déboursés budgétaires et donnent lieu à des déficits budgétaires et donc à l'augmentation de la dette publique.

\section{4 - L'ultime intervention : les banques poubelles}

Plusieurs proposent la création de banques poubelles ou de « bads banks » comme moyen de sortie de crise (le Forum économique de Davos, le Président Obama). La raison d'être d'une bad bank est de permettre aux établissements financiers de se débarrasser des prêts ou des titres qui minent la qualité de leurs actifs et de leur redonner le goût de prêter une fois pour toutes.

L'idée n'est pas nouvelle. Déjà utilisée dans l'Empire Romain et à Florence à la Renaissance, cette technique a été à l'honneur dans un passé plus récent. Lors de la « crise de l'endettement » des pays du Tiers monde, dans les années 1980, les grandes banques ont assaini leur bilan. À cette époque, un marché secondaire des mauvaises créances des pays du Tiers monde a été mis en place. Les banques mirent aux enchères des titres, trop risqués à leurs yeux, qu'un plus grand preneur de risques pouvait acheter avec une décote. Par exemple, un titre d'une valeur nominale de 100 millions de dollars était vendu à 70 millions, donc avec une décote de $30 \%$. La banque vendeuse inscrivait une recette de 70 millions et une perte de 30 millions. L'acheteur détenait une créance de 100 millions payée 70 millions. La banque se retrouvait avec un actif plus "sain » avec une perte déductible de l'impôt. Le pays du Tiers monde avait toujours une dette de 100 millions. L'idée des banques poubelles est du même ordre.

Il s’agit de créer un établissement public-privé disposé à acheter à escompte des créances jugées trop risquées par les banques. En nettoyant ainsi l'actif de ces entreprises, l'objectif est de réduire leurs craintes de défaillances de leurs débiteurs pour qu'elles recommencent à prêter. L'estimation de la valeur de ces titres serait l'ordre de 1000 à 2000 milliards. La banque poubelle aura sans doute des pertes, mais certaines créances seront partiellement ou totalement honorées. En fin de compte, l'établissement public-privé devrait arriver à l'équilibre. Sinon, l'État assumera les pertes. Le plan Obama prévoit des revers pouvant aller jusqu’à 500 milliards.

La restructuration des papiers commerciaux adossés à des actifs (PCAA) est semblable. La stratégie ici est de geler à long terme des titres à court terme, généralement à trois mois. L'espoir est que, dans plusieurs années, la perte sur le PCAA soit le plus 
faible possible. Les pertes seront assumées par le gouvernement fédéral et ceux du Québec, de la Colombie-Britannique et de l'Ontario. Le problème des détenteurs de ces papiers commerciaux (Caisse de dépôt et placement du Québec, Jean Coutu, Transat, Aéroports de Montréal, etc) est de voir des fonds, qu'ils croyaient à court terme, être immobilisés à long terme.

En plus de ces tentatives de relance du crédit, il faut maintenant enrayer les conséquences de la crise de la sphère financière sur la sphère de production, sur le secteur réel. L’Europe, la Chine, l'Australie, les États-Unis et même le Canada ont tous adopté des plans budgétaires de relance. Les dépenses des gouvernements, beaucoup dans les infrastructures, visent à stimuler l'économie pour suppléer aux manquements des établissements financiers. Dans ces programmes, une large place est laissée aux réductions d'impôts pour contenter les visions des conservateurs (voir la partie I du texte et dans celui de Pierre-Antoine Harvey). Aux États-Unis, le Président Obama pour faire adopter son plan a dû faire d'importantes concessions aux sénateurs républicains et à la droite démocrate, 286 milliards de baisse d'impôt, le tiers du programme.

\section{2 - Colmater le système financier : les mesures envisagées à moyen terme}

La conférence du Groupe des $20^{3}$ (ou du G-20) du 15 novembre 2008 est parvenue à une entente de principe sur le constat de la crise financière et de la nécessité d'agir pour empêcher sa répétition. En plus de favoriser des mesures budgétaires pour stimuler la demande interne et de condamner le protectionnisme, le G-20 concluait qu'il fallait accroître la coopération économique, améliorer l'évaluation des risques financiers, des normes comptables et la surveillance des marchés financiers.

Sous la pression des grands pays «émergents » (Inde, Chine, Brésil, Afrique du Sud), il a été convenu d'une plus grande " ouverture » aux pays émergents. Pour les États-Unis et l'Europe partager le pouvoir n'est pas très naturel. Comment pourrontils perdre leur droit de veto dans les grands organismes internationaux ? L'ordre mondial peut-il être modifié ?

3 Ces pays réalisent les 2/3 du commerce mondial et 90\% du PIB de la planète. 
La prochaine rencontre du G-20 se déroulera à Londres à partir du 2 avril où les ministres des finances devront soumettre un plan d'une nouvelle architecture financière internationale qui a été discuté les 14 et 15 février à Rome. De ce qui a pu filtrer, rien de fondamental ne sera remis en cause. Encore une fois, il ne s'agira que de renforcer les normes prudentielles déjà en vigueur. Essentiellement, et encore une fois, on remettra à jour les normes du Comité de Bâle de la Banque des Règlements Internationaux ${ }^{4}$ (la BRI). Comme les trois dernières normes (Bâle I, II et III) adoptées depuis 35 ans. Ces codes de bonne conduite n’ont eu aucune efficacité et n’en auront pas plus sans mesures coercitives puissantes.

\section{B - CE QU'IL FAUT PLUTÔT FAIRE !}

Compte tenu des fonds engloutis dans les banques par les gouvernements et le rôle essentiel du crédit dans nos économies, les banques devraient être nationalisées, comme les compagnies d'assurance. Mais remettons ce projet pour l'instant et limitons nous à une vision réformiste.

D'une part, nous ne pouvons remettre en selle le système financier pour lui laisser faire ce qu'il veut, même mieux encadré. D’autre part, notre modèle économique, basé sur la consommation sans frein et le gaspillage des ressources, ne peut plus être la référence au développement. Ce modèle n'est pas exportable pour la survie de la planète. Pour un véritable changement, il faut aller au fond des choses.

\section{1 - Réguler la finance}

La cause fondamentale de la crise financière que nous vivons est la conséquence de la mutation du modèle de l'activité bancaire traditionnelle vers un nouveau modèle basé sur la non-responsabilisation des acteurs. En effet, l'ancien modèle impliquait

4 La Banque des Règlements Internationaux (BRI) est une organisation internationale crée en 1930 qui œuvre à la coopération monétaire et financière internationale et fait office de banque des banques centrales. Son siège social est à Bâle (Suisse) et le capital de la BRI est détenu exclusivement par des banques centrales (55), dont la Banque du Canada. 
que l'établissement financier qui accordait un crédit portait les risques de cette décision jusqu'au remboursement final. La banque avait donc tout intérêt à s'assurer de la solvabilité de son client du début à la fin du processus.

Alors que le modèle qui nous a conduit à la crise actuelle permet à l'établissement financier initiateur du crédit de se débarrasser des risques en les vendant après titrisation. Le crédit accordé (hypothèque, cartes de crédit, prêts automobiles, obligations d'entreprises, etc.) est transformé en titre financier et est vendu sur les marchés financiers, comme les PCAA. Les nouveaux acheteurs peuvent faire de même. Ainsi de titrisations en titrisations le contrôle du premier débiteur et la responsabilité du premier prêteur disparaissent ${ }^{5}$. Ces montages financiers souvent très complexes sont le fruit d'une ingénierie financière de plus en plus imaginative. Rapidement, on ne sait plus sur quoi reposent ces titres, l'identité et la solvabilité du premier bénéficiaire du crédit ne sont plus connues, ni les suivants d'ailleurs. À la moindre défaillance, l'édifice s'écroule. Ce fut le cas des subprimes (les prêts hypothécaires à haut risque) avec l'arrêt du gonflement de la bulle immobilière. Dans ce cirque, les agences de notation ont été les complices de ces pratiques d'opacité des marchés et ont incité tous les établissements financiers à y plonger pour satisfaire leur cupidité.

De certaines mesures urgentes :

a. Les établissements financiers ne doivent plus pouvoir prêter n'importe comment et à n'importe qui sans tenir compte des risques. La responsabilité de faire face aux risques inhérents à tout prêt accordé doit relever de l’initiateur du crédit et être connu.

b. La titrisation devrait être limitée à deux niveaux et chaque crédit ainsi transformé devrait être identifié, autant au niveau du débiteur et que du créditeur.

c. Les transactions anonymes ou cachées sur les marchés financiers devraient être interdites. En particulier les opérations de gré à gré sur les produits dérivés par les entreprises.

5 La titrisation existe depuis plus de trente ans, mais elle s'est systématisé dans les années 1990 et 2000. 
d. De a, b et c, limiter tout crédit pour fins spéculatives. Que le niveau de risque de chaque crédit soit connu et évalué par un organisme de notation indépendant.

e. Dans une véritable volonté de contrôle, les paradis fiscaux doivent se plier aux règles ou disparaître. C'est la fin des paradis.

f. Des normes éthiques : la rémunération des dirigeants doit être responsable. Les primes ne seraient encaissables qu'aux cinq ans et que partiellement (50\%). En cas de mauvaise performance, les primes pourraient être remboursées. Aucune prime ne devrait exister en cas de départ volontaire ou involontaire. La pratique des remises d'options d'actions aux cadres devrait être abolie. Cette pratique a amené des dérives préjudiciables aussi bien aux actionnaires qu'aux travailleurs.

g. La gouvernance de la finance mondialisée ne peut être que mondiale. L'application de ces mesures, et de bien d'autres ${ }^{6}$, sera sous la responsabilité d'une organisation internationale relevant des États, du politique.

\section{2 - Redéfinir notre modèle économique}

Sur ce sujet, je vous invite à lire l'excellent texte de Gilles Bourque « $\underline{\text { Pour un }}$ modèle québécois plus durable » sur le site d’économie autrement.

Fin du texte

6 Comme le contrôle des marchés des changes et de la circulation des capitaux à court terme, qui sont essentiellement des opérations spéculatives. 